\title{
Biomarker Potential of Urine miR-451 at Different Stages of Diabetic Nephropathy
}

Emel Isiktas Sayilar ${ }^{1 *}$, Mustafa Gullulu ${ }^{1}$, Ercan Tuncel ${ }^{2}$, Hande Peynirci $^{2}$, Adem Alemdar $^{3}$, Berrin Tunca $^{3}$, Unal Egeli ${ }^{3}$, Gulsah Cecener ${ }^{3}$, Murat

Bayindir $^{4}$ and Gokhan Cosgun ${ }^{4}$

${ }^{1}$ Departments of Nephrology, Uludag University School of Medicine, Bursa, Turkey

${ }^{2}$ Endocrinology and Metabolism, Uludag University School of Medicine, Bursa, Turkey

${ }^{3}$ Medical Biology, Uludag University School of Medicine, Bursa, Turkey

${ }^{4}$ Internal Medicine, Uludag University School of Medicine, Bursa, Turkey

\begin{abstract}
Aims: To evaluate the potential of urinary miR-451 as a biomarker at different stages of diabetic nephropathy.

Methods: A total of 45 subjects having stage 3 chronic kidney disease $(n=15)$ or stage 5 chronic kidney disease $(n=15)$ and 15 healthy volunteers were included. Data on patient demographics, laboratory findings [creatinine, estimated glomerular filtration rate, urinary protein excretion] target genes and functions of the selected MicroRNAs associated with diabetic nephropathy and fold differences in the level of MicroRNA expression in blood and urine and the correlation of urine and plasma MicroRNA expression with estimated glomerular filtration rate were recorded.

Results: MiR-195 expression level among stage 3 chronic kidney disease patients was higher in plasma samples compared to the control group, while it was significantly lower in the urine samples $(p=0.036)$. In the stage 5 chronic kidney disease patient group, while the expression level was significantly higher in the plasma samples $(p=0.005)$, urine sample expression was lower but not significantly different than the control group. Compared to the controls, miR-451 expression level was higher in the plasma samples of stage 3 chronic kidney disease patients, but significantly lower in the urine samples $(p=0.019)$. Among the stage 5 chronic kidney disease patients, there was significantly higher level of expression in plasma samples $(p=0.007)$ and significantly lower expression in urine samples $(p=0.022)$ than the control group.
\end{abstract}

Conclusions: Our study is original with its investigation of MicroRNA expressions at different stages of chronic kidney disease. Especially the statistically significant changes in the expression of miR-195 and miR-451 make these MicroRNAs come forward as good noninvasive biomarker candidates.

Keywords: Chronic kidney disease; MicroRNA expression; Plasma; Urine; Diabetic nephropathy

\section{Introduction}

Diabetes mellitus (DM), with increasing prevalence rates, causes microvascular complications such as nephropathy, retinopathy, and neuropathy and macrovascular complications such as coronary artery disease, atherosclerosis, hypertension and stroke [1]. Many factors play a role in the development of complications. Increased oxidative stress due to hyperglycemia, advanced glycation end products (AGEs), protein kinase $\mathrm{C}$ (PKC) and mitogen-activated protein kinase (MAPK) activity increase secretion of cytokines, chemokines, angiotensin II, and transforming growth factor- $\beta 1$ (TGF- $\beta 1$ ) $[2,3]$. Among these, TGF$\beta 1$ leads to diabetic nephropathy (DN) development by increasing profibrotic gene expression [4,5]. While hyperfiltration and mesangial hypertrophy develop at early stages of DN, basal membrane thickening, podocyte dysfunction, proteinuria, tubulointerstitial fibrosis, and glomerulosclerosis develop later in the process [6,7].

$\mathrm{DN}$ is the most common cause of end-stage renal failure worldwide. Early diagnosis of DN reduces renal complication risk and mortality and morbidity rates of the patients. Though microalbuminuria is the standard test used for the early stage diagnosis of $\mathrm{DN}$, there are many studies reporting it as inadequate [8]. Since microalbuminuria is evaluated via immunoassay method, only the immunoreactive form of albumin can be measured [9]. Over the recent years, new techniques and biomarkers have been adopted for early stage diagnosis and progression evaluation of $\mathrm{DN}$.

MicroRNAs (miRNA) are single stranded RNA molecules with 20 to 23 nucleotides in length, taking place in each step of the cell cycle but protein coding. They display their functions at the posttranscriptional stage as a negative regulator in the series specific modulation of gene expression. In the biogenesis steps of miRNA, first the primary transcript pri-miRNA is formed by RNA polymerase II (RNA Pol II) in the nucleus. Then, pri-miRNA is sliced by Drosha and Pasha to form pre-miRNA that is the precursor molecule. Pre-miRNAs are transferred to cytosol via the nuclear membrane protein "exportin-5". The premiRNA is sliced by the transactivation-responsive RNA binding protein (TARBP) and Dicer in the cytoplasm and forms short RNA. Of the short RNAs, double stranded molecules are formed, one containing the guide miRNA and the other containing the series conjugated to the guide miRNA. The guide strand of the double-stranded molecule is combined with the RNA induced silencing complex (RISC) and causes the targeted messenger-RNA (mRNA) cleavage or blocks its translation. miRNAs bind with the 3'UTR sequence of one or more

*Corresponding author: Emel Isiktas Sayilar, Department of Nephrology, Uludag University School of Medicine, Bursa Turkey, Tel: +90 507964 80 90; Fax: +90 224 29514 44; E-mail: emelisiktas@yahoo.com

Received February 06, 2016; Accepted February 19, 2016; Published February 25, 2016

Citation: Sayilar El, Gullulu M, Tuncel E, Peynirci H, Alemdar A, et al. (2016) Biomarker Potential of Urine miR-451 at Different Stages of Diabetic Nephropathy. J Diabetes Metab 7: 650. doi:10.4172/2155-6156.1000650

Copyright: $\odot 2016$ Sayilar El, et al. This is an open-access article distributed under the terms of the Creative Commons Attribution License, which permits unrestricted use, distribution, and reproduction in any medium, provided the original author and source are credited. 
mRNAs with partial or full complementarity [10]. miRNAs are involved as regulators in cell proliferation, differentiation, apoptosis, neoplastic transformation, and apoptosis. miRNAs, unlike mRNAs, having a highly stable structure, have been used as a potential clinical biomarker as they are easily obtained from primarily blood and also urine, saliva, amniotic fluid, breast milk, sweat, seminal fluid and tissues [11,12]. Today, approximately 28 miRNA that are directly related to pathogenesis of DN are defined [13].

miRNAs contribute to DN development via multiple paths. There are studies from recent years that evaluate the level of renal fibrosis by miRNA gene expression analysis [14]. According to the hypotheses of these studies, miRNAs lead to renal fibrosis by causing changes in TGF- $\beta$, extracellular matrix and epithelial-mesenchymal transformation $[15,16]$.

Among these, TGF- $\beta 1$ that causes proliferation in extracellular matrix, fibroblast, and myofibroblasts has the most important role [17]. Number of TGF- $\beta 1$ receptors increase with renal damage, activating the TGF- $\beta /$ Smad 3 pathway and fibrosis is initiated [18]. In vitro studies show that miR-192 increases TGF- $\beta 1$ levels and regulation of other miRNAs, and causes hypertrophy, glomerular expansion and fibrosis in renal mesangial cells $[19,20]$. Recently, miR-192 level in the urine sample and proteinuria were compared in the prediction of interstitial fibrosis, and the miR-192 level was observed to be a good indicator [2123]. Putta et al. have shown that renal fibrosis and proteinuria can be reduced by specifically inhibiting miR-192 [24].

miR-21 is one of the miRNAs that often has increased expression in solid organ tumors [25]. Studies also showed that miR-21 plays a role in DN pathogenesis [26,27]. Increased miR-21 expression inhibits renal mesangial cell proliferation via PTEN/PI3K/Akt pathway and takes on a preventative role against nephropathy development by reducing glomerular hypertrophy and albuminuria [28].

It has been shown that miR-195 can play a role in fibrosis via pathways that are effective on both endothelial (EndMT) and epithelial (EMT) mesenchymal transformation (microRNAs in kidney fibrosis 2013). It is known to be effective on elements such as TNF and SMAD3 in TGF- $\beta 1$ signal pathway, and on Myc, TP53 and SMAD3 in Wnt signal pathway. Its suppressing effect on SMAD3 in both pathways gives way to the thought that it may be more effective on fibrosis [29].

In addition to the signal pathways that play a role in EMT, miR451 has also been shown to play a role in inflammation-related fibrosis [30]. It has also been found to increase fibrosis in other tissues via TNF, SMAD3, and AMH genes in TGF- $\beta 1$ pathway that is another fibrosis mechanism.

Increased expression of miR-124 has been shown to cause podocyte secretion by reducing the podocyte uptake capacity in streptozotocininduced DN in Winstar rats. Under mechanic stress, miR-124 is believed to target INTEGRIN $\alpha 3 \beta 1$, which is a cell-matrix binding receptor [31].

Use of miRNA analyses in plasma and urine samples as a biomarker in diabetic complications is important, because they can facilitate early diagnoses of both the diseases and the complications. By modifying the treatment and taking the necessary precautions, patient survival and quality of life can be increased. Additionally, the less yielding nature of miRNAs to decomposition and features such as being non-invasive and easy to collect make it more likely to be preferred as a biomarker. Despite this potential, there are very few studies that demonstrate use of urine miRNA level measurement in the diagnoses and monitoring of DN today [23].
In this study, we investigated the important roles of miRNAs in $\mathrm{DN}$ pathogenesis and their use as a biomarker in the evaluation of DN prognosis via urine and plasma level measurements.

\section{Materials and Methods}

A total of 45 subjects were included in this study, who presented at the Uludag University's Nephrology and Endocrinology Departments between July 2013 and March 2014. Among the participants, 15 had stage 3 chronic kidney disease (CKD), 15 had stage 5 CKD, and 15 were healthy volunteers. The study was conducted after the approval of the Clinical Research Ethics Committee of Uludag University School of Medicine (2013-12/22). Demographic data of the patients were recorded from the medical history and electronic files. All procedures followed were in accordance with the ethical standards of the responsible committee on human experimentation (institutional and national) and with the Helsinki Declaration of 1975, as revised in 2008. Informed consent was obtained from all patients for being included in the study.

The participant inclusion criteria included being at the age of 1875 years of; having DM and hypertension for a minimum of 5 years, systolic blood pressure $\leq 140 \mathrm{mmHg}$ or diastolic blood pressure $\leq$ $90 \mathrm{mmHg}$ under antihypertensive treatment. Patients with active infection symptoms, malignancy diagnosis, kidney disease associated with renal vascular disease, glomerulonephritis, polycystic kidney diseases, anatomic abnormality associated with the urinary system or an endocrine disease other than DM; who were under peritoneal dialysis or hemodialysis treatment or pregnant were excluded from the study.

Blood pressure measurements were done by using an Erka (P.M.S Instruments Ltd, Berkshire, United Kingdom) sphygmomanometer following a minimum of a 5-minute rest from the right arm, in a seated position. Venous blood samples were collected into tubes containing ethylenediaminetetraacetic acid and were stored at $-80^{\circ} \mathrm{C}$. Urine samples were transferred to the laboratory immediately after collection and were stored at $-80^{\circ} \mathrm{C}$ until the miRNA isolation procedure. Glomerular filtration rate (GFR) of the patients was calculated using the modification of diet in renal disease (MDRD, $\mathrm{mL} / \mathrm{min} / 1.73 \mathrm{~m}^{2}$ ) formula [32]. Laboratory parameters such as blood hemoglobin, urea, creatinine, electrolyte, albumin and parathyroid hormone levels and 24hour urinary protein excretion were obtained from the electronic records.

\section{RNA extraction and quantification}

miRNA isolation from the plasma samples was performed by Qiagen miRNA Easy kit and from the urine samples by Norgen Urine microRNA purification kit, following the manufacturers' instructions. miRNA concentrations and purity were determined with NanoDrop 2000 UV spectrophotometer (Thermo Scientific, USA) using the A260 / $280 \mathrm{~nm}$ ratio.

\section{Reverse transcriptase-polymerase chain reaction}

Comparative reverse transcriptase-polymerase chain reaction (RTPCR) procedure with triplicate samples and 5 miRNAs with RNU6B as the endogenous control was performed by using LightCycler 480II device (Roche Diagnostics, USA) in the presence of negative control. The relative expressions of miRNAs were calculated using the $\mathrm{Ct}$ method. miRNA fold differences in the samples with different DN stages compared to controls were calculated using $2^{-\Delta \Delta \mathrm{Ct}}$ method.

\section{Determination of miRNA Targets}

miRWALK online database was used to determine the miRNA 
Citation: Sayilar El, Gullulu M, Tuncel E, Peynirci H, Alemdar A, et al. (2016) Biomarker Potential of Urine miR-451 at Different Stages of Diabetic Nephropathy. J Diabetes Metab 7: 650. doi:10.4172/2155-6156.1000650

target genes (Table 1) [13]. miRWALK, by using KEDD and BioCArta pathway databases, covers information on gene functions as well.

\section{Statistical Analysis}

Sabioscience online calculation program was used for miRNA expression analyses [33]. For other statistical analyses, GraphPad Prism version 6 (GraphPad Software, La Jolla California USA) was used.

\section{Results}

\section{Clinical Data}

A total of 45 volunteer participants were included in the study.
There were 11 females and four males among the 15 stage 3 CKD patients, whose median age was 63.07 (range: 33-74) years. Among the stage 5 CKD patients, four were female and 11 were male, and their median age was 59 (range: $34-75$ ). Of the 15 healthy volunteers with GFR $>60 \mathrm{~mL} / \mathrm{min}, 11$ were female and four were male, and their median age was 41 (range: $35-46$ ) years.

Demographic characteristics and laboratory results of the patients are displayed in Table 2; and the fold differences of miRNA expressions in blood and urine compared to the control group are presented in Table 3.

Comparison of the miR-21 expression levels with the control

\begin{tabular}{|c|c|}
\hline miRNA ID & miRNA accession number \\
\hline hsa-mir-21 & MI0000077 \\
\hline hsa-mir-195 & \\
\hline hsa-mir-451 & \\
\hline & \\
\hline & \\
\hline
\end{tabular}

Target genes and functions of selected miRNAs related with DN MAPK3 member of the MAP kinase family

MYC plays a role in cell cycle progression, apoptosis and cellular transformation SMAD2

SMAD3 functions as a transcriptional modulator activated by transforming growth factor-beta SMAD7 is a nuclear protein that binds the E3 ubiquitin ligase SMURF2

SMAD3 functions as a transcriptional modulator activated by transforming growth factor-beta TGFB1 regulate proliferation, differentiation, adhesion, migration, and other functions in many cell types CCND1 function as regulators of CDK kinases

APC a tumor suppressor protein that acts as an antagonist of the Wnt signaling pathway JUN interacts directly with specific target DNA sequences to regulate gene expression WNT1 functions in the induction of the mesencephalon and cerebellum RELA NFKB2 bound to either REL, RELA

TLR4 plays a fundamental role in pathogen recognition NFKB1 stimulates the expression of genes involved in a wide variety of biological functions TNF involved in the regulation of a wide spectrum of biological processes including cell proliferation, differentiation SMAD3 functions as a transcriptional modulator activated by transforming growth factor-beta MYC plays a role in cell cycle progression, apoptosis and cellular transformation CCND1 function as regulators of CDK kinases

MAPK8 involved in a wide variety of cellular processes such as proliferation, differentiation

TP53 a tumor suppressor protein containing transcriptional activation, DNA binding TLR4 plays a fundamental role in pathogen recognition

NFKB1 stimulates the expression of genes involved in a wide variety of biological functions

SMAD3 functions as a transcriptional modulator activated by transforming growth factor-beta TNF involved in the regulation of a wide spectrum of biological processes including cell proliferation, differentiation AMH is a member of the transforming growth factor-beta gene family

MAPK9 involved in cellular processes such as proliferation, differentiation, transcription regulation and development

Table 1: The target genes and functions of the selected miRNAs associated with diabetic neuropathy (DN).

\begin{tabular}{|c|c|c|c|}
\hline & Control (n=15) & Stage 3 CKD $(n=15)$ & Stage 5 CKD $(n=15)$ \\
\hline \multirow[t]{2}{*}{ Gender (female / male) } & $11 / 4$ & $11 / 4$ & $4 / 11$ \\
\hline & \multicolumn{3}{|c|}{ Mean \pm SEM } \\
\hline Age (years) & $41.0 \pm 0.8$ & $63.1 \pm 3.0$ & $59.0 \pm 3.2$ \\
\hline Creatinine (mg/dL) & $0.73 \pm 0.04$ & $1.83 \pm 0.22$ & $4.66 \pm 0.65$ \\
\hline eGFR (mL/min) & $109.7 \pm 18.1$ & $36.0 \pm 10.5$ & $14.3 \pm 9.6$ \\
\hline Hemoglobin (g/dL) & $13.1 \pm 0.4$ & $12.3 \pm 0.4$ & $10.9 \pm 0.4$ \\
\hline Albumin (g/dL) & $4.2 \pm 0.2$ & $3.6 \pm 0.5$ & $3.4 \pm 0.5$ \\
\hline Urinary protein excretion (g/day) & $0.2 \pm 0.01$ & $2.9 \pm 0.5$ & $3.5 \pm 0.3$ \\
\hline
\end{tabular}

Table 2: Demographic characteristics and laboratory findings of the patients.

\begin{tabular}{|c|c|c|c|c|c|c|c|c|}
\hline & \multicolumn{4}{|c|}{ Urine } & \multicolumn{4}{|c|}{ Plasma } \\
\hline & \multicolumn{2}{|c|}{ Stage 3 CKD } & \multicolumn{2}{|c|}{ Stage 5 CKD } & \multicolumn{2}{|c|}{ Stage 3 CKD } & \multicolumn{2}{|c|}{ Stage 5 CKD } \\
\hline & Fold difference & $P$ value & Fold difference & $P$ value & Fold difference & $P$ value & Fold difference & $P$ value \\
\hline \multicolumn{9}{|l|}{ RNA U6* } \\
\hline Mir-192 & 3.25 & 0.119 & 1.84 & 0.145 & 3.76 & 0.114 & -1.48 & 0.684 \\
\hline Mir-195 & -1.09 & 0.036 & -2.97 & 0.112 & 22.86 & 0.076 & 34.28 & 0.006 \\
\hline Mir-21 & 1.54 & 0.524 & 1.03 & 0.218 & 7.05 & 0.220 & 6.58 & $0.060^{* *}$ \\
\hline Mir-124 & 84.09 & 0.212 & 38.58 & 0.228 & -6.24 & 0.483 & -40.45 & 0.299 \\
\hline Mir-451 & -4.07 & 0.019 & -9.51 & 0.003 & 34.31 & 0.075 & 15.47 & 0.007 \\
\hline
\end{tabular}

Table 3: Fold differences in the level of miRNA expression in plasma and urine in stage 3 and 5 chronic kidney disease (CKD) patients compared to the control group. 
Citation: Sayilar El, Gullulu M, Tuncel E, Peynirci H, Alemdar A, et al. (2016) Biomarker Potential of Urine miR-451 at Different Stages of Diabetic Nephropathy. J Diabetes Metab 7: 650. doi:10.4172/2155-6156.1000650

Page 4 of 6

group showed that plasma and urine expressions were higher, not significantly, by 7.0 and 1.5 fold, respectively among stage $3 \mathrm{CKD}$ patients; and although the fold changes were similar to stage $3 \mathrm{CKD}$ patients, 6.6 fold increase in the plasma expression was found to be marginally significant $(\mathrm{p}=0.06)$, while it was not in the urine samples (1.0 fold) of the stage 5 CKD patients.

Compared to the control group, significantly higher levels of MiR124 expression in the urine samples of stage 3 and 5 CKD patients $(84.1$ and 38.6 fold, respectively) were observed, while the difference was lower among stage 5 patients than in stage 3 patients. An evaluation of the plasma levels yielded that the expression was lower among stage 3 and 5 patients compared to the control group ( -6.3 and -40.5 fold, respectively) and the expression decreased by the increase in CKD stage.

In terms of miR-192 expression levels, stage $3 \mathrm{CKD}$ patients had similarly higher fold differences in plasma and urine samples (3.8 and 3.3 fold, respectively) compared to the control group. Stage $5 \mathrm{CKD}$ patients, on the other hand, had a lower plasma (-1.5 fold) and a higher urine (1.8 fold) expression of miR-192 which were not significantly different than the control group.

miR-195 expression level among stage 3 CKD patients was higher in plasma samples compared to the control group ( 22.9 fold; $\mathrm{p}=0.075$ ) while it was significantly lower in the urine samples ( -1.1 fold; $\mathrm{p}=0.036$ ). In the stage $5 \mathrm{CKD}$ patient group, while the expression level was significantly higher in the plasma samples ( 34.3 fold; $\mathrm{p}=0.005)$, urine sample expression was lower $(-2.97$ fold; $\mathrm{p}=0.111)$ but not significantly different than the control group.

Compared to the controls, miR-451 expression level was higher in the plasma samples of stage $3 \mathrm{CKD}$ patients ( 34.3 fold; $\mathrm{p}=0.075$ ), but significantly lower in the urine samples ( -4.1 fold; $p=0.019$ ). Among the stage $5 \mathrm{CKD}$ patients, there was significantly higher level of expression in plasma samples ( 15.5 fold; $\mathrm{p}=0.007)$ and significantly lower expression in urine samples ( -9.5 fold; $\mathrm{p}=0.022)$ than the control group.

\section{Clinical correlations}

We reviewed the correlations between the selected miRNAs with significant or marginally significant plasma and urine expression levels and eGFR in patients with CKD associated with diabetic and hypertensive nephropathy (Figures 1 and 2).

The plasma miR-124 expression was lower among the stage 3 CKD patients compared to the control group, while higher levels were observed in miR-21 miR-192, miR-195, and most markedly in miR-451 expressions; while in urine samples, significantly lower levels in miR195 and miR-451 expressions, and higher levels in miR-21, miR-124, and miR-192 of expressions were detected (Figure 3).

Among the stage $5 \mathrm{CKD}$ patients, plasma samples showed insignificantly higher miR-21 expression, significantly higher miR-195 and miR-451 expressions, and lower miR-124 and miR-192 expressions. Their urine samples showed increased miR-21, miR-124, and miR-192 expressions, decreased miR-195 expression and significantly higher miR-451 expression (Figure 3).

\section{Discussion}

Diabetic and hypertensive nephropathy is the most common cause of end-stage renal failure (ESRF) worldwide. Early diagnosis of nephropathy reduces the risk of ESRF and leads to decrease in diseaserelated mortality and morbidity rates. There is a need for easy-to-apply, non-invasive, fast and reliable biomarkers for early diagnosis and studies on miRNA expression analyses have been bringing hope to the field.

The main factors that define the miRNA expression profile are hyperglycemia, inflammatory cytokines, proteinuria, advanced age, high blood pressure, and hypoxia. miR-192, miR-194, miR-204, miR215 and miR-216 among the miRNAs have been shown to be more

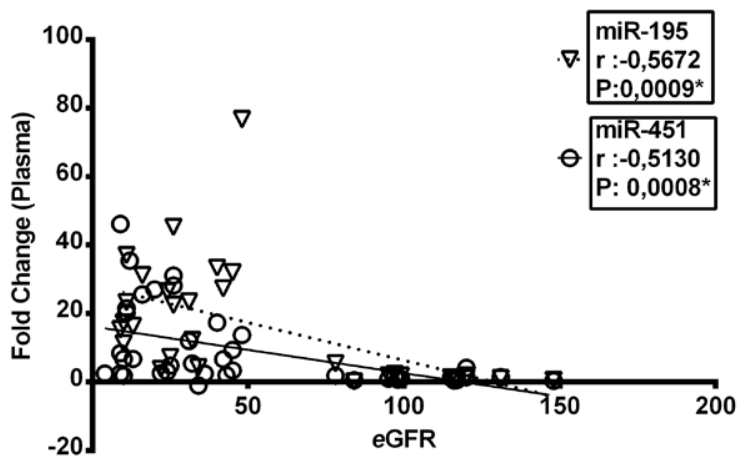

Figure 1: Correlations between estimated glomerular filtration rate (eGFR) and plasma miRNA expressions.

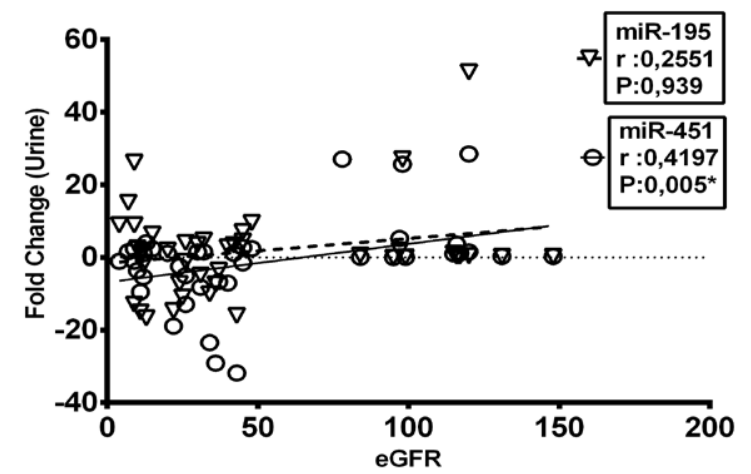

Figure 2: Correlations between estimated glomerular filtration rate (eGFR) and urine miRNA expressions.

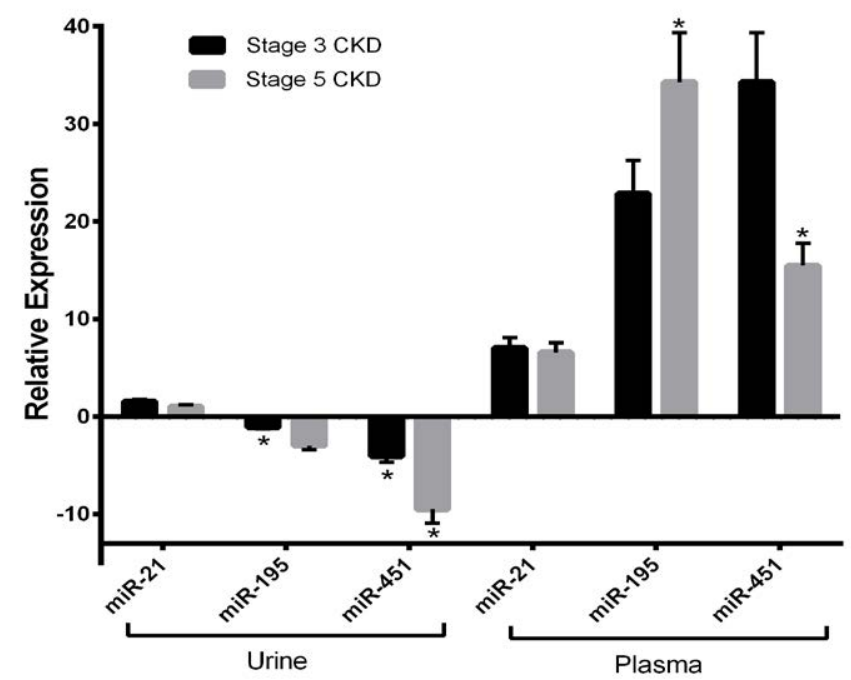

Figure 3: Plasma and urine miRNA expression levels among stage 3 and 5 chronic kidney disease (CKD) patients. 
expressed in renal fibrosis development [34]. Kato et al.'s [35] rat model study showed increased miR-192 expression in diabetic rats and increased TGF-1 levels in mesangial cells. Krupa et al.'s [36] study demonstrated tubulointerstitial fibrosis and GFR-related decreases in miR-195 expression among patients with advanced DN. In Neal et al.'s study, all miRNA levels were decreased in parallel to the renal functions among patients with severe renal failure [37]. miRNAs are excreted into urine via glomerular ultrafiltrate or tubular secretion. Melkonyan et al.'s study determined 22 different miRNAs in the urine, none of which were kidney-specific, and all were demonstrated to have reached the kidney via blood circulation and were filtrated through the glomeruli [38]. There are studies demonstrating that miR-192 decreases with increased fibrosis and that miR-192 is reversely proportional to the severity of DN as well [36, 39]. Similarly, in our study, compared to the control group, miR-192 expression was 1.5 fold lower in plasma and 1.8 fold higher in urine samples of stage 5 CKD patients, while it was much higher in both plasma and urine samples (3.8 and 3.3 fold, respectively) of stage $3 \mathrm{CKD}$ patients.

In vivo and in vitro studies conducted at early stages of DN showed that miR-21 inhibited mesangial proliferation via PTEN/ PI3K/Akt pathway. At first, hypertrophy is tried to be overcome in the glomeruli and the mesangial area by increasing miR-21 expression but as DN progresses, proteinuria and fibrosis develop. Zhang et al. administered miR-21 to cell lines and diabetic rats and demonstrated an increase in urine albumin excretion by the end of four weeks [40]. In a more recent study based on the hypothesis that DN develops in association with miR-21 expression in $\mathrm{db} / \mathrm{db}$ rats, it was emphasized that suppression of TGF- $\beta$ and NF- $\mathrm{kB}$ pathways by miR-21 may affect fibrosis and inflammation, respectively; and that suppression of Smad7 can affect kidney damage [27]. In our study, both stage 3 and stage 5 CKD patients had higher miR-21 expression in both plasma and urine samples compared to controls but this difference was higher among the stage 3 patients most probably in association with degree of fibrosis and hypertrophy.

miR-195, a member of the miRNA family that play a role in DN etiology and physiology, show antiapoptotic features due to its down-regulation. Chen et al.'s [41] rat model study showed that in early stages of $\mathrm{DN}$, miR-195 expression decreased, was along with a reverse association between miR-195 expression level and glomerular diameter. Higher than control urinary and plasma miR-195 expression levels in stage 3 and stage 5 CKD patients, respectively in the present study can be explained by the furthering of the renal parenchymal damage and primarily renal cortex damage in relation with miR-195 down-regulation; shrinking in the glomeruli; fibrosis; and increased apoptosis. Moreover, recent studies also showed that high glucose concentration could elevate miR-195 expression [42,43].

Over-expression of miR-451 in DN inhibits glomerular and mesangial cell proliferation both in vivo and in vitro [44]. During early stages of DN, miR-451 expression decreases and glomerular hypertrophy and mesangial proliferation develop. It has been demonstrated that with miR-451, Ywhaz is targeted and mesangial hypertrophy in earlier stages of DN is prevented via p38 MAPK signal pathway. In our study, while the plasma level was higher, urine level was significantly lower than controls among stage $3 \mathrm{CKD}$ patients. In case of stage 5 CKD patients, compared to stage 3 group, both plasma and urine miR-451 expression levels were lower at a statistically significant level. This finding matches with that of Calida et al.'s study that reported decreased overall plasma miRNA levels in CKD [32]. Similarly, a study conducted on kidney tissue reported higher expression of miR-451 in renal cortex of hypertensive patients compared to normotensive subjects [45]. The higher levels of miR-451 expression in plasma and tissue compared to normal individuals and the low expression levels in urine samples makes us believe that the miR-451 in the urine may be associated with DN. The significant correlation of the decrease in miR-451 expression with eGFR values may be explained with this miRNA preventing glomerular hypertrophy. The decreased miR-451 expression causes increased activity of signal pathways such as MAPK9 and SMAD3 and is believed to increase hypertrophy and therefore development of DN.

\section{Conclusion}

Past studies have used urine and plasma samples from a very low number of patients. Often the studies were conducted in rats and at cell lines. Our study is original with its investigation of miRNA expressions at different stages of CKD. Especially the statistically significant changes in the expression of miR-195 and miR-451 make these miRNAs come forward as good noninvasive biomarker candidates. The utility of these miRNAs in the clinical practice, whose mechanisms of actions have been discovered, should be investigated with larger-scale studies.

\section{Author's Contributions}

AA carried out the molecular genetic studies, participated in the sequence alignment and drafted the manuscript. GC and UE carried out the immunoassays. BT participated in the sequence alignment. EIS and HP participated in the design of the study and performed the statistical analysis. MB and GC conceived of the study, and participated in its design and coordination. MG and ET have given final approval of the version to be published. All authors read and approved the final manuscript.

\section{Acknowledgements}

This study was conducted with support of Bursa Kidney Foundation.

\section{References}

1. Beckman JA, Creager MA, Libby $P$ (2002) Diabetes and atherosclerosis: epidemiology, pathophysiology, and management. JAMA 287: 2570-2581.

2. Kim W, Hudson BI, Moser B, Guo J, Rong LL, et al. (2005) Receptor for advanced glycation end products and its ligands: a journey from the complications of diabetes to its pathogenesis. Ann N Y Acad Sci 1043: 553-561.

3. Brownlee M (2005) The pathobiology of diabetic complications: a unifying mechanism. Diabetes 54: 1615-1625.

4. Zhu Y, Usui HK, Sharma K (2007) Regulation of transforming growth factor beta in diabetic nephropathy: implications for treatment. Semin Nephrol 27: 153-160.

5. Kato M, Castro NE, Natarajan R (2013) MicroRNAs: potential mediators and biomarkers of diabetic complications. Free Radic Biol Med 64: 85-94.

6. Ziyadeh FN, Sharma K (2003) Overview: combating diabetic nephropathy. J Am Soc Nephrol 14: 1355-1357.

7. Mason RM, Wahab NA (2003) Extracellular matrix metabolism in diabetic nephropathy. J Am Soc Nephrol 14: 1358-1373.

8. Jin J, Ku YH, Kim Y, Kim Y, Kim K, et al. (2012) Differential proteome profiling using ITRAQ in microalbuminuric and normoalbuminuric type 2 diabetic patients. Exp Diabetes Res 2012: 168602.

9. Liu R, Li G, Cui XF, Zhang DL, Yang QH, et al. (2011) Methodological evaluation and comparison of five urinary albumin measurements. J Clin Lab Anal 25: 324-329.

10. Shomron N, Levy C (2009) MicroRNA-biogenesis and Pre-mRNA splicing crosstalk. J Biomed Biotechnol 2009: 594678.

11. Mitchell PS, Parkin RK, Kroh EM, Fritz BR, Wyman SK, et al. (2008) Circulating microRNAs as stable blood-based markers for cancer detection. Proc Nat Acad Sci U S A 105: 10513-10518.

12. Cortez MA, Bueso-Ramos C, Ferdin J, Lopez-Berestein G, Sood AK, et al 
Citation: Sayilar El, Gullulu M, Tuncel E, Peynirci H, Alemdar A, et al. (2016) Biomarker Potential of Urine miR-451 at Different Stages of Diabetic Nephropathy. J Diabetes Metab 7: 650. doi:10.4172/2155-6156.1000650

(2011) MicroRNAs in body fluids--the mix of hormones and biomarkers. Nat Rev Clin Oncol 8: 467-477.

13. Holistic view of validated disease-miRNA interactions.

14. Tesch GH (2010) Review: Serum and urine biomarkers of kidney disease: A pathophysiological perspective. Nephrology (Carlton) 15: 609-616.

15. Conway B, Hughes J (2012) Cellular orchestrators of renal fibrosis. QJM 105 611-615

16. Kato M, Park JT, Natarajan R (2012) MicroRNAs and the glomerulus. Exp Cell Res 318: 993-1000.

17. Lan HY (2011) Diverse roles of TGF- $\beta^{2} /$ Smads in renal fibrosis and inflammation. Int J Biol Sci 7: 1056-1067.

18. Meng XM, Chung AC, Lan HY (2013) Role of the TGF- $\beta^{2} / B M P-7 / S m a d$ pathways in renal diseases. Clin Sci (Lond) 124: 243-254.

19. Kato M, Natarajan R (2012) MicroRNA circuits in transforming growth factor- $\beta^{2}$ actions and diabetic nephropathy. Semin Nephrol 32: 253-260.

20. Deshpande SD, Putta S, Wang M, Lai JY, Bitzer M, et al. (2013) Transforming growth factor- $\beta^{2}$-induced cross talk between p53 and a microRNA in the pathogenesis of diabetic nephropathy. Diabetes 62: 3151-3162.

21. Szeto CC, Ching-Ha KB, Ka-Bik L, Mac-Moune LF, Cheung-Lung CP, et al. (2012) Micro-RNA expression in the urinary sediment of patients with chronic kidney diseases. Dis Markers 33: 137-144.

22. Wang G, Kwan BC, Lai FM, Chow KM, Li PK, et al. (2013) Urinary sediment miRNA levels in adult nephrotic syndrome. Clin Chim Acta 418: 5-11.

23. Yang Y, Xiao L, Li J, Kanwar YS, Liu F, et al. (2013) Urine miRNAs: potentia biomarkers for monitoring progression of early stages of diabetic nephropathy. Med Hypotheses 81: 274-278.

24. Putta S, Lanting L, Sun G, Lawson G, Kato M, et al. (2012) Inhibiting microRNA-192 ameliorates renal fibrosis in diabetic nephropathy. J Am Soc Nephrol 23: 458-469.

25. Chow TF, Youssef YM, Lianidou E, Romaschin AD, Honey RJ, et al. (2010) Differential expression profiling of microRNAs and their potential involvement in renal cell carcinoma pathogenesis. Clin Biochem 43: 150-158.

26. Dey N, Das F, Mariappan MM, Mandal CC, Ghosh-Choudhury N, et al. (2011) MicroRNA-21 orchestrates high glucose-induced signals to TOR complex 1 resulting in renal cell pathology in diabetes. J Biol Chem 286: 25586-25603.

27. Zhong X, Chung AC, Chen HY, Dong Y, Meng XM, et al. (2013) miR-21 is a key therapeutic target for renal injury in a mouse model of type 2 diabetes. Diabetologia 56: 663-674

28. Mahimainathan L, Das F, Venkatesan B, Choudhury GG (2006) Mesangial cell hypertrophy by high glucose is mediated by downregulation of the tumor suppressor PTEN. Diabetes 55: 2115-2125.

29. Nigam V, Sievers HH, Jensen BC, Sier HA, Simpson PC, et al. (2010) Altered microRNAs in bicuspid aortic valve: a comparison between stenotic and insufficient valves. J Heart Valve Dis 19: 459-465.

30. Rosenberger CM, Podyminogin RL, Navarro G, Zhao GW, Askovich PS, et al. (2012)
miR-451 regulates dendritic cell cytokine responses to influenza infection. J Immunol 189: 5965-5975.

31. Li D, Lu Z, Jia J, Zheng Z, Lin S (2013) MiR-124 is related to podocytic adhesive capacity damage in STZ-induced uninephrectomized diabetic rats. Kidney Blood Press Res 37: 422-431.

32. Levey AS, Bosch JP, Lewis JB, Greene T, Rogers N, et al. (1999) A more accurate method to estimate glomerular filtration rate from serum creatinine: a new prediction equation. Modification of Diet in Renal Disease Study Group. Ann Intern Med 130: 461-470.

33. RTA 2 PCR Array Data Analysis.

34. Srivastava SP, Koya D, Kanasaki K (2013) MicroRNAs in kidney fibrosis and diabetic nephropathy: roles on EMT and EndMT. Biomed Res Int 2013: 125469

35. Kato M, Zhang J, Wang M, Lanting L, Yuan H, et al. (2007) MicroRNA-192 in diabetic kidney glomeruli and its function in TGF-beta-induced collagen expression via inhibition of E-box repressors. Proc Natl Acad Sci U S A 104 3432-3437.

36. Krupa A, Jenkins R, Luo DD, Lewis A, Phillips A, et al. (2010) Loss of MicroRNA-192 promotes fibrogenesis in diabetic nephropathy. J Am Soc Nephrol 21: 438-447.

37. Neal CS, Michael MZ, Pimlott LK, Yong TY, Li JY, et al. (2011) Circulating microRNA expression is reduced in chronic kidney disease. Nephrol Dial Transplant 26: 3794-3802.

38. Melkonyan HS, Feaver WJ, Meyer E, Scheinker V Shekhtman EM, et al (2008) Transrenal nucleic acids: from proof of principle to clinical tests. Ann N Y Acad Sci 1137: 73-81.

39. Wang B, Herman-Edelstein M, Koh P, Burns W, Jandeleit-Dahm K, et al. (2010) E-cadherin expression is regulated by miR-192/215 by a mechanism that is independent of the profibrotic effects of transforming growth factor-beta. Diabetes 59:1794-1802.

40. Zhang Z, Peng H, Chen J, Chen X, Han F, et al. (2009) MicroRNA-21 protects from mesangial cell proliferation induced by diabetic nephropathy in $\mathrm{db} / \mathrm{db}$ mice. FEBS Lett 583: 2009-2014

41. Chen YQ, Wang XX, Yao XM, Zhang DL, Yang XF, et al. (2012) Abated microRNA-195 expression protected mesangial cells from apoptosis in early diabetic renal injury in mice. J Nephrol 25: 566-576.

42. Chen YQ, Wang XX, Yao XM, Zhang DL, Yang XF, et al. (2011) MicroRNA-195 promotes apoptosis in mouse podocytes via enhanced caspase activity driven by BCL2 insufficiency. Am J Nephrol 34: 549-559.

43. Mortuza R, Feng B, Chakrabarti S (2014) miR-195 regulates SIRT1-mediated changes in diabetic retinopathy. Diabetologia 57: 1037-1046.

44. Zhang Z, Luo X, Ding S, Chen J, Chen T, et al. (2012) MicroRNA-451 regulates p38 MAPK signaling by targeting of Ywhaz and suppresses the mesangia hypertrophy in early diabetic nephropathy. FEBS Lett 586: 20-26.

45. Marquez FZ, Campain AE, Tomaszewski M, Zukowska-Szczechowska E, Yang $\mathrm{YH}$, et al. (2011) Gene expression profiling reveals renin mRNA overexpression in human hypertensive kidneys and a role for microRNAs. Hypertension 58 1093-1098. 